\title{
An injection optimization decision-making tool using streamline based fuzzy logic workflow
}

\author{
Nader Y. BuKhamseen ${ }^{1}\left[\right.$ D Abdullah A. Al-Najem ${ }^{1}$ - Ali H. Saffar ${ }^{1}$
}

Received: 25 May 2021 / Accepted: 23 October 2021 / Published online: 9 November 2021

(c) The Author(s) 2021

\begin{abstract}
This paper presents an adaptive approach to optimize field injection strategies using streamline-based well allocations coupled with fuzzy logic. The strength of our approach comes from the fact that streamlines are generated by running a full-physics reservoir simulator. Streamlines provide great insights about well pattern connectivity and good allocation factors allowing the injection efficiency (IE) for each pattern to be determined. Fuzzy logic can simulate human thinking and handle different categories of information including linguistic, imprecise, approximate, and overlapping to name a few. This paper presents a genuine approach for field injection optimization using a streamlined-based fuzzy logic system. In this work, we present an adaptive streamline-based fuzzy logic system that uses three input parameters namely injection efficiency (IE), water cut (WC), and injection loss to aquifer to assign an injector ranking index (IRI) according to injector performances. The workflow then smartly redistributes water injection by accounting for operational constraints and number of connected producers in a pattern in addition to the IRI. The workflow examines the low performers (i.e., low and medium IRI categories) and assigns different injection reduction factors for each injector in these categories. Then, the total amount of reduced injection is assigned to high performers (i.e., high IRI) while ensuring no operational constraint is violated, such as bottom-hole pressure (BHP) and capacity of pumps. This approach has been tested on a dual-porosity dual-permeability (DPDP) conceptual simulation model. The area of interest has two rows of injectors: downdip and updip. The updip injectors are the focus of the study. The results of applying this approach show noticeable improvements in injection efficiency for most wells in the area of interest ensuring better sweep, good pressure support, and improving cumulative oil production. We believe combining both technologies, namely streamlines and fuzzy logic, can provide reservoir engineers with a robust decision-making tool to attain a more successful field-wide water injection strategy.
\end{abstract}

Keywords Streamlines $\cdot$ Water injection $\cdot$ Fuzzy logic

\section{Introduction}

Numerous challenges are associated with fields under continuous water injection. Therefore, proactive water management practices and continuous optimization are necessary. An important key to attaining these objectives is establishing a better understanding of the relationship between injectors and producers.

While (Bouaouaja et al. 2012) conventionally determined producer/injector interrelationships based on constant (i.e., fixed) predefined patterns, several authors successfully

Nader Y. BuKhamseen

nader.bukhamseen@aramco.com

1 Saudi Aramco: Saudi Arabian Oil Co, Dhahran, Saudi Arabia utilized streamlines technology in fieldscale studies and demonstrated a more accurate representation of the dynamic irrigation/drainage regions within the reservoir (Thiele and Batycky 2003; Spyrou et al. 2017; Liu et al. 2010). For example, Thiele et al. (2010) used streamlines to provide great insights about the strength of connectivity between wells and paths followed by water to connected producers as shown in Fig. 1.

Full-field injection optimization is a complex task requiring continuous injection rate adjustments of many injectors simultaneously. Therefore, an intelligent method is needed to assist with this endeavor.

Streamlines are instantaneous lines tangential to the velocity field obtained from pressure potential field (DattaGupta and King 2007; Thiele et al. 2010) as illustrated in Fig. 2. Streamlines are projected from injectors to producers 


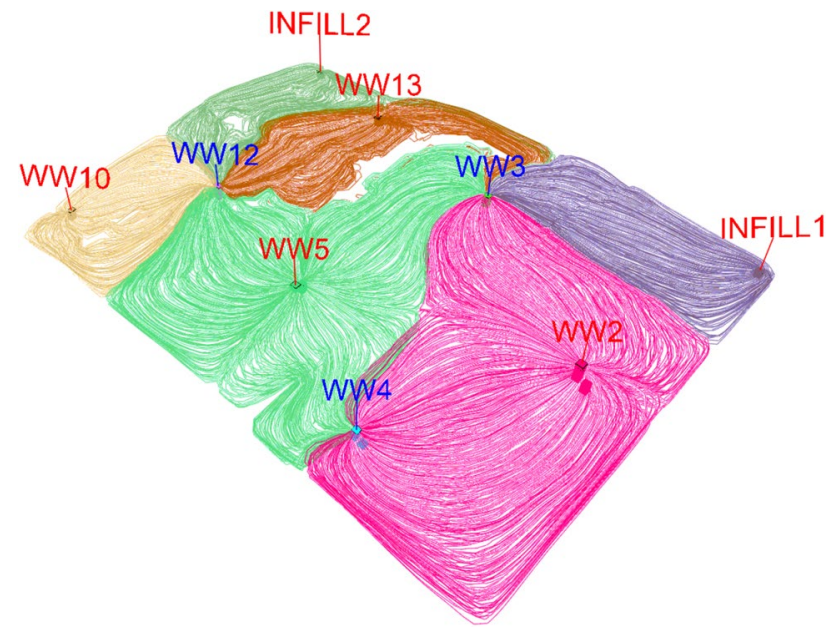

Fig. 1 Using streamlines to map producer/injector connectivity [Source: Thiele et al. (2010)]

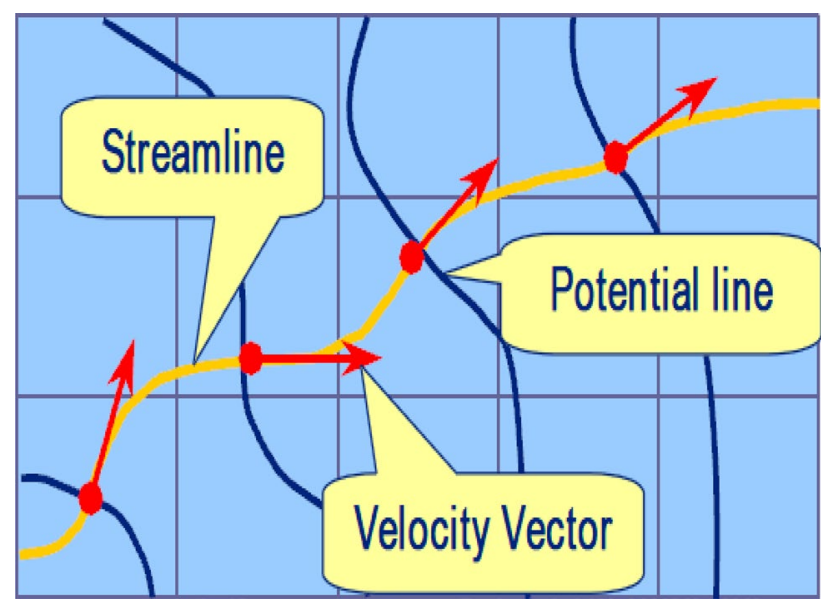

Fig. 2 Streamline tracing and velocity vector mapping (Ibrahim et al. 2007)

allowing the calculation of well allocation factors (WAF), i.e., the amount of fluid supporting any producer in a pattern as shown in Eq. (1) (Thiele and Batycky 2004). It is worth mentioning that the numerator of Eq. (1) represents the summation of all streamline fluxes associated with each pair.

$\mathrm{WAF}^{p-i}=\frac{\sum_{s=1}^{n_{s l}} Q^{p-i}}{Q^{p}} \times 100$

where $Q p-i$ is oil production rate from producer $p$ being supported by injector $i$,

$Q p$ is the total oil production rate from producer $p$,

$n s l$ is the number of streamlines connecting producer $p$ to injector $i$.

A proprietary simulator has been enhanced to trace streamlines using fluxes computed as part of finite difference flow simulation. In addition, streamline quantities such as WAFs and injection efficiency (IE), which is the ratio of produced oil to injected water of each producer/injector pair (Kornberger et al. 2014), are obtained and made readily available for engineers to analyze injection performance (Al-Zawawi et al. 2011).

By coupling these streamlined quantities with fuzzy logic, a robust decision-making tool has been designed to help engineers implement efficient and optimized injection strategies. The prowess of fuzzy logic is that it simulates human thinking. It can handle different categories of information such as linguistic, imprecise, approximate, and overlapping (Widarsono et al. 2005). This makes fuzzy logic a useful tool for engineers to use for decision-making.

The concept of fuzzy set theory was developed by Zadeh in 1965 (Zadeh 1965). In the conventional set theory, an element either completely belongs or does not belong to a set (Boolean). In the fuzzy set theory, on the other hand, an element can partially belong to a set through a degree of membership. The three basic components of a fuzzy system are fuzzy variables, membership functions, and fuzzy rules. Fuzzy variables are the input factors to be considered in making a decision or output. Membership functions define the degree of truth of the fuzzy variable in the fuzzy set. Fuzzy rules describe the relationship between input and output in forms of fuzzy IF-THEN rules.

Fuzzy logic has been applied in the oil and gas industry for a number of years. Applications include petrophysics, reservoir characterization, enhanced recovery, well stimulation, and infill drilling (Mohaghegh 2000). In this paper, we present a genuine approach to optimize fieldwide injection strategies using stream-line information coupled with fuzzy logic. Our approach captures the ever-changing flow paths and injector/producer connectivity especially in cases of new drilling and shut-in of wells (BuKhamseen et al. 2016, 2017). Results show noticeable improvements in injection efficiency for most wells while honoring best practices in reservoir management.

\section{Workflow description}

The general workflow of our approach is depicted in Fig. 3a. It starts by running the full-physics simulator base case with the option of generating streamlines. The workflow is terminated if the field is under-injecting or injection efficiencies (IE) are already optimal (e.g., IE for all injectors $>$ preset value). If the previous conditions are not met, streamlines are analyzed to evaluate the spatial and temporal performance of different injection patterns.

Fuzzy logic's membership functions for IE, water cut (WC), and injection loss are constructed. Fuzzy logic rules are then applied to the combination of input parameters to 


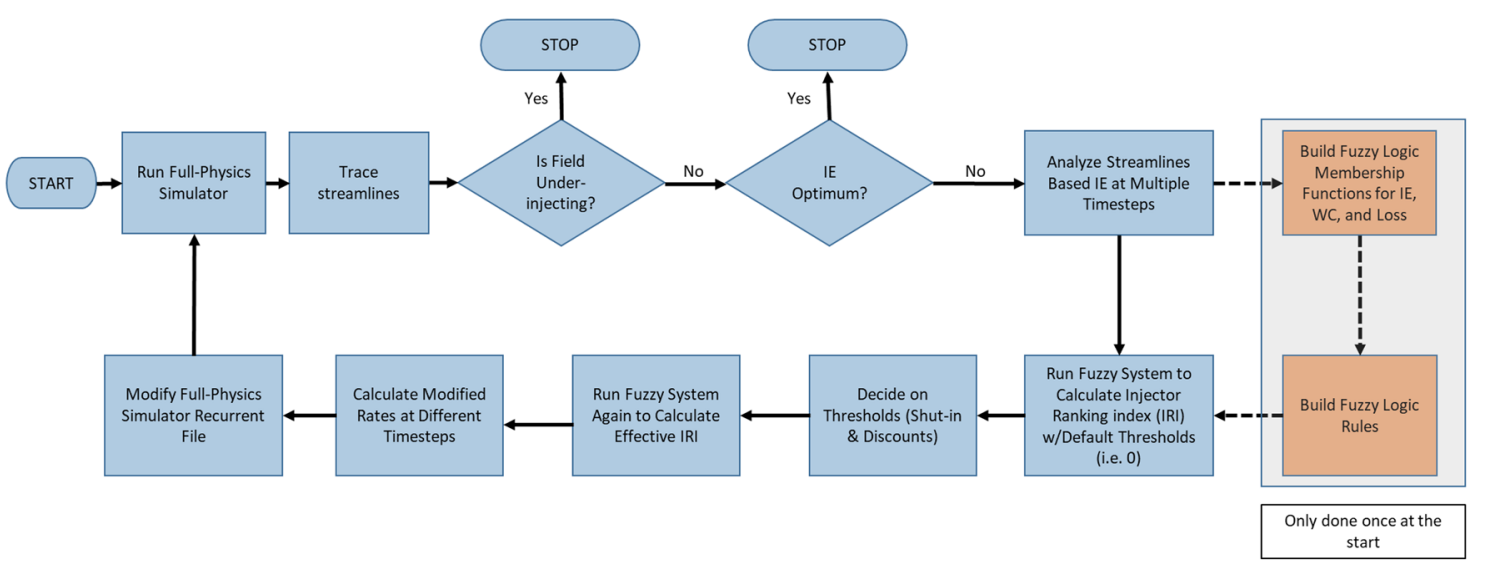

a The workflow of the streamline-fuzzy logic system to optimize injection strategy

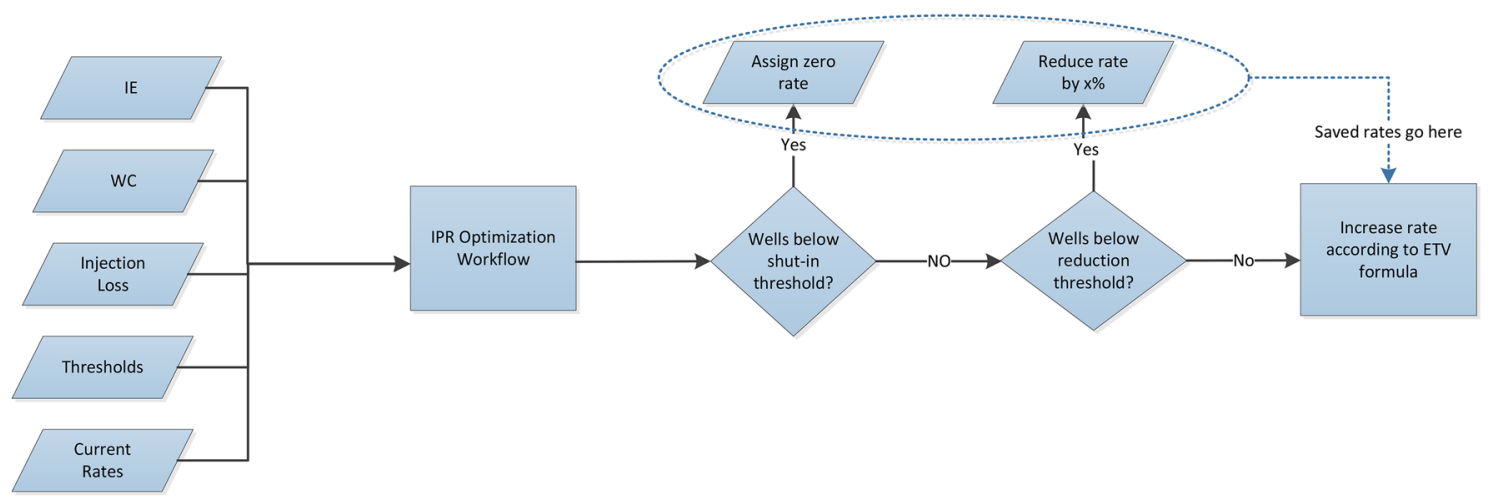

b Detailed workflow of assigning new injection rates

Fig. 3 a The workflow of the streamline-fuzzy logic system to optimize injection strategy. b Detailed workflow of assigning new injection rates

produce injector ranking indices (IRI). The IRI is basically a measure of how the input parameters (IE, WC, injection loss) impact the performance of each injector. Each injector is then classified into low, medium, and high based on their IRIs. The higher the IRI is, the more optimized the injection rate is. On the contrary, low IRI indicates the need for further optimization.

Inefficient injectors are shut-in based on their IRIs rather than on injection efficiency alone. Thresholds to define shutin wells and rate cutbacks are applied to inefficient injector and avail the spared amount of injection volumes to the more efficient injectors. Consequently, we introduced the effective injector ranking index (eIRI). This concept more sensibly accounts for the water injection capacity of each injector and is calculated based on the estimated true value (ETV) as shown in Fig. 3b. The concept of ETV is inspired by Google Analytics' weighted sort algorithm and is explained by Meyers (2010).

The ETV helps quantify the influence of the value of one factor on another. The IRI value is the outcome of the defuzzification process that resulted from the composition of fuzzy rules. It is represented as a crisp value. In our case, the normalized IRI is influenced by the injection capacity of each injector resulting in the effective IRI (eIRI) that is calculated using the following:

$\operatorname{eIRI}=\left(Q_{i} / Q_{i-\max }\right) * \operatorname{IRIi}+\left(1-\left(Q_{i} / Q_{i}-\max \right)\right) * \operatorname{IRI}_{\text {avg }}$

$Q_{i}$ : current injection rate for each injector.

$Q_{i \text {-max }}:$ maximum injection rate of all injectors.

IRIi: current normalized IRI for each injector.

IRI $_{\text {avg: }}$ : average normalized IRI for all injectors.

The eIRI is then used to calculate the new injection rate of the high efficient injectors as follows

$Q_{\text {new_efficientInjector }}=\left(\right.$ eIRIi $/ \sum$ eIRI $) *$ Spared Volume $+Q i$

The optimal IE referred to in the second validation step in Fig. 3a is a result of a simple maximization problem. 
We start at a base value of IE, and then we apply all the fuzzy rules and update the injection rates. These rates are then fed to the simulator and a new IE is calculated from the new run. The process continues until there is no further incremental improvement in IE. Moreover, the optimization process could be dynamically included within the workflow using any maximization algorithm such as gradient-based approach.

Figure 4 shows the example of three good injectors and how spared volume may be distributed differently using weighted average method compared to our workflow. The weighted average assigns rate based on injection capacity

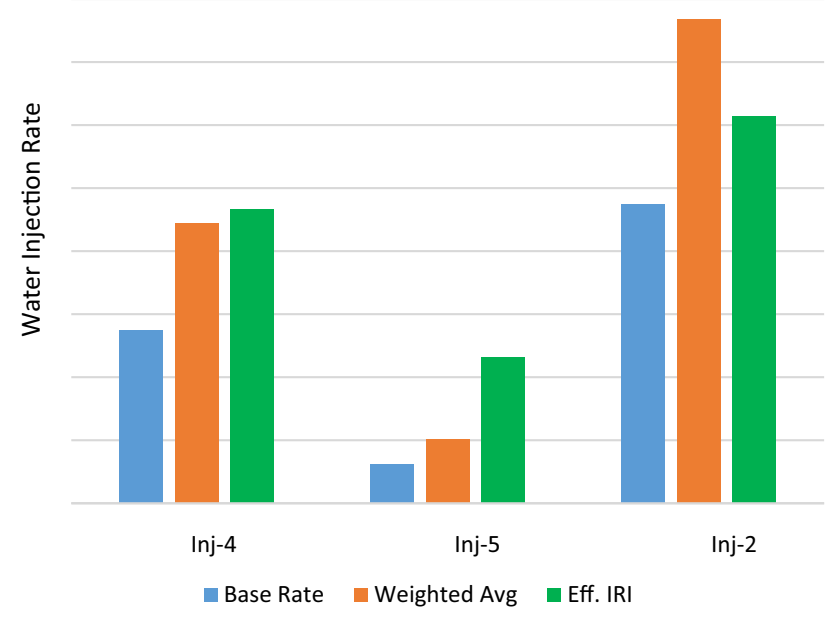

Fig. 4 Water injection rates comparison among different injectors showing the difference in rate distribution between the weighted average and eIRI only whereas our workflow takes into consideration both the injection capacity as well as the IRI. For instance, due to having a high IRI, the effective IRI suggests that injector 2 receive more water volume than that assigned by the weighted average method. Injector 4 , with middle-range IRI, receives less water volume from the effective IRI method than the weighted average method despite having an injection capacity higher than injector 2 .

Figure 5 illustrates the modifications to the water injection rates suggested by our streamlines-fuzzy logic system based on the calculations of the eIRI. We see here that different actions were taken at different times during future forecasts. For example, during the first two timesteps, the injector received additional injection rate. During the following time step (i.e., time $=3$ ) the rate was reduced to reflect the decline in injection efficiency of this specific injector. Another great example that demonstrates the continuous assessment by our workflow happened at time $=4$ where additional rate was assigned to the same injector, which probably is due to the addition of new producer in the irrigation area of this injector. Interestingly, the same injector was shut-in by our workflow at time $=6$ probably due to water recycling, dead or shut-in producers.

\section{Application}

The fuzzy logic model encompasses decision rules and semantic queries. It is a knowledge repository in the form of rules and relationships created by subject matter experts and reservoir engineers based on the underlying experience and detailed understanding of the asset. Since the simulation model adequately captures the subsurface and operational
Fig. 5 Example of the impact of implementing the workflow on the water injection rate

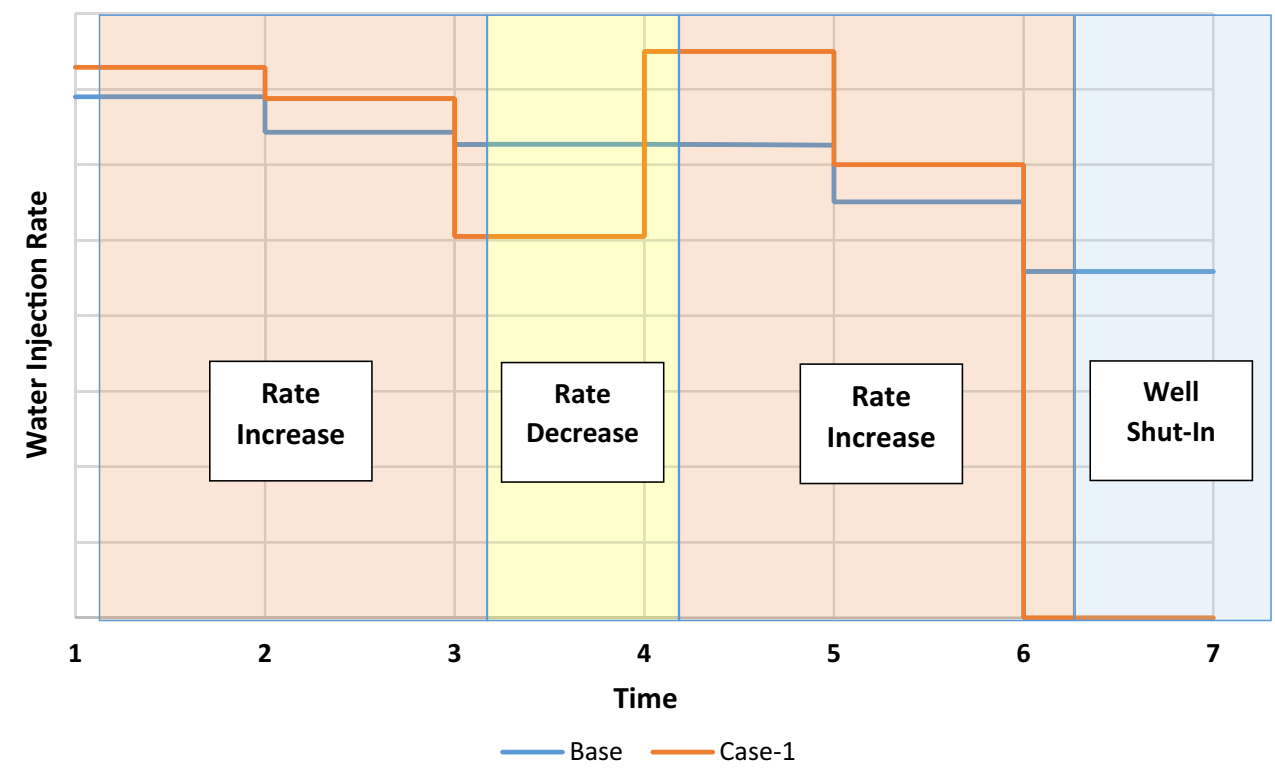


uncertainty, the fuzzy logic model then operates within the realm of that uncertainty.

The optimized streamlines-fuzzy logic workflow was tested on a conceptual model in a swept region with high water production. There are two sets of injectors: seven in the north and seven in the south. This was necessary to properly maintain the required amount of water injection in each sub-region and continuously improve the connectivity between injectors and producers. Additionally, to ensure adequate pressure support, reducing total water injection was not considered in this case. As a result, all optimization scenarios presented here are based on smartly re-distributing the injection volume saved out of the inefficient injectors.

In this model application, the optimization process started by running the base case and reviewing the injection efficiency of all injectors in the two sets. This step is a prerequisite for designing the proper fuzzy logic membership functions. In our case, three fuzzy logic's membership functions are constructed for injection efficiency (IE), water cut (WC), and injection loss to aquifer (Loss). Fuzzy logic rules are then applied to the combination of input parameters to produce injector ranking indices (IRI). The IRI is basically a measure of how the input parameters (IE, WC, injection loss) impact the performance of each injector. Each injector is then classified into low, medium, and high based on their IRIs. The higher the IRI is, the more optimized the injection
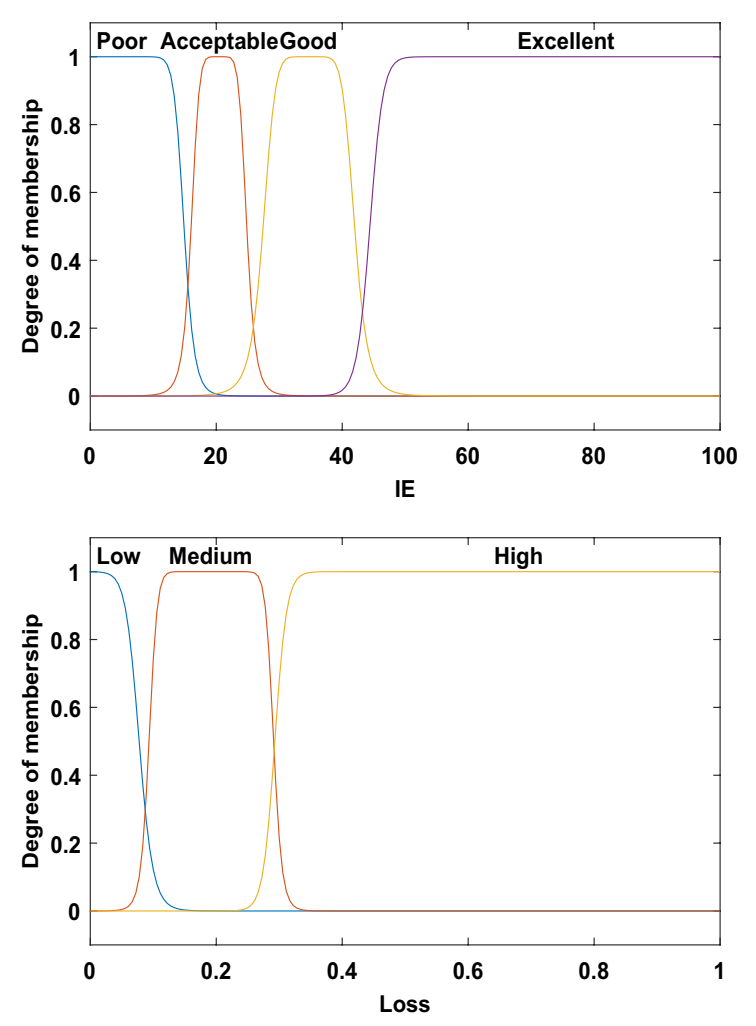

rate is. On the contrary, low IRI indicates the need for further optimization.

Figure 6 shows the membership functions of the three input parameters to the streamlines-fuzzy logic namely injection efficiency (IE), water cut (WC), and injection loss to aquifer (Loss) as well as the injector ranking index (IRI), which is the output of the fuzzy logic. Additionally, Table 1

Table 1 Input and Output Variables

\begin{tabular}{lll}
\hline Input variable & Fuzzy set name & Range \\
\hline Injection Efficiency IE & Poor & Less than 20 \\
& Acceptable & 10 to 30 \\
& Good & 20 to 50 \\
& Excellent & Greater than 40 \\
Water Cut WC & Low & Less than 30 \\
& Medium & 20 to 60 \\
Injection Loss & High & Greater than 50 \\
& Low & Less than 0.18 \\
& Medium & 0.10 to 0.35 \\
Injector Ranking Index IRI & High & Greater than 0.25 \\
& Medium & Less than 35 \\
& High & 30 to 65 \\
& & Greater than 58 \\
\hline
\end{tabular}
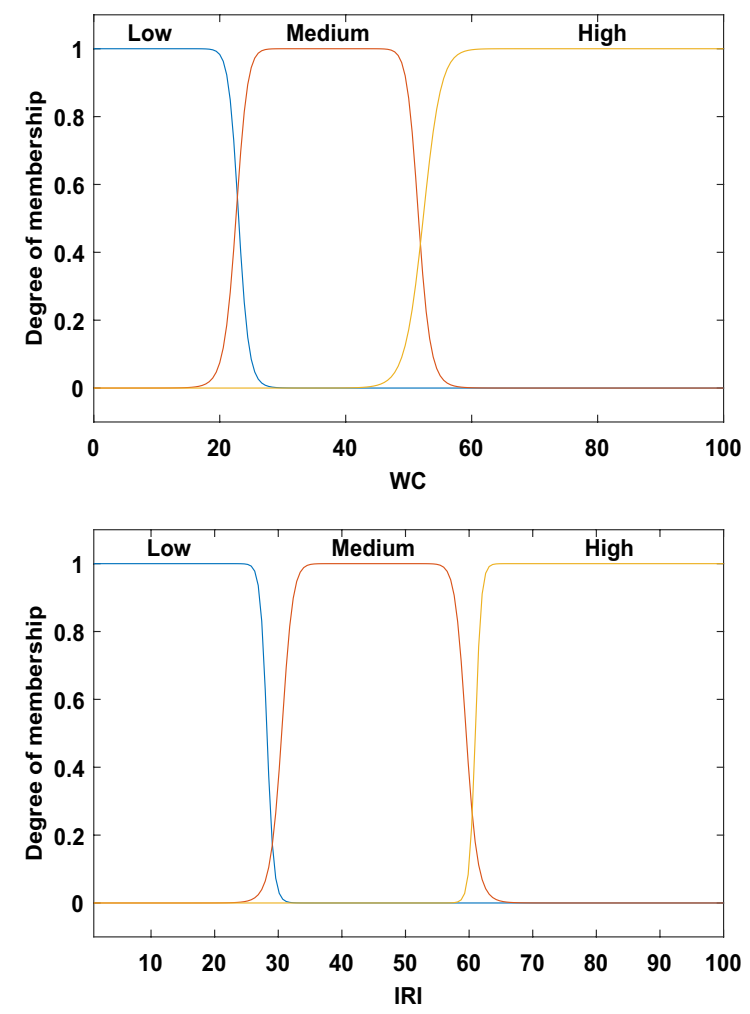

Fig. 6 The membership functions used in the fuzzy logic system 
summarizes the fuzzy set names of the input and output parameters and their ranges.

There are different stages of fuzzy control as indicated by Fig. 7. The first stage is fuzzification where input variables are assigned degrees of membership in various classes. Fuzzification process then maps the inputs to values from 0 to 1 using a set of membership functions. Figure 8 indicates that a crisp value of $I E=25$ is mapped to a value of 0.55 for the 'Good' class of Injection Efficiency.

The second stage is rule evaluation where inputs are applied to a set of if/then rules such as:

If (IE is Acceptable) and (WC is Medium) and (Loss is High) then (IRI is Medium).

The results of several rules are aggregated together to generate a set of fuzzy outputs. The final stage is defuzzification. Figure 9 shows an example of how operators work in rule composition. In this example, it is assumed that $\mathrm{IE}=20$, $\mathrm{WC}=53$, and Loss $=0.5$. The "AND" operator is applied in all calculations, which implies taking the minimum values of the membership functions. The defuzzification value denoted as $X^{*}$ uses the centroid method:

$X^{*}=\frac{\sum_{i=1}^{n} x_{i} \cdot \mu\left(x_{i}\right)}{\sum_{i=1}^{n} \mu\left(x_{i}\right)}$

where $x_{i}$ indicates the sample element, $\mu\left(x_{i}\right)$ is the membership function, and $\mathrm{n}$ represents the number of elements in the sample. The resultant IRI value of 73 is an outcome of the defuzzification process using the centroid formula as shown in Fig. 10.

Different sensitivity runs were made to determine the best threshold and rate discount combination that maximizes our objective of optimizing oil production in this case. Figure 11 shows a histogram of the computed IRI from the fuzzy logic system for all the injectors from multiple timesteps. In this example, injectors with an IRI less than 25 will be shutin. Additionally, the injection rate of injectors with an IRI

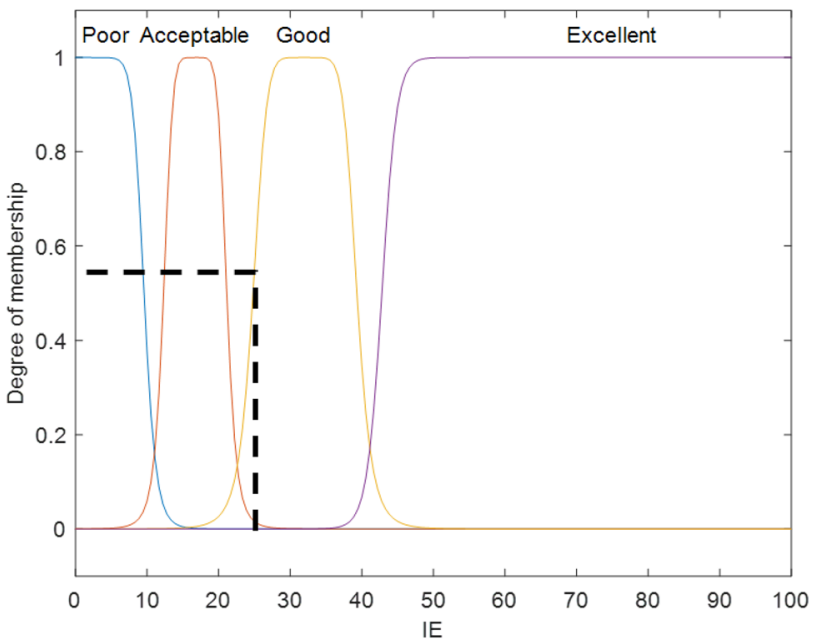

Fig. 8 Determination of the output of a membership function

between 25 and 34 will be reduced by $20 \%$. Similarly, if the threshold is between 35 and 40, the injection rates will be reduced by $10 \%$. Finally, injectors with IRI greater than 40 receive additional rates saved from low IRI injectors (i.e., below 40) as calculated by the ETV formula. Another example with the same thresholds but different discount factors (i.e., 30 and 20\%) was tested. As anticipated, this scenario would provide extra water volume compared to the past example.

It is very important to carry some sensitivity runs to help decide on the optimum injection rate of each injector. Figure 12 shows an example (Case-1 and 2) of the sensitivity runs we conducted to compare the effect of different thresholds and discount factors. As we see here, this high IRI injector received additional water volume in Case- 2 compared to Case-1 in times 3, 4, and 6. This happens as a result of applying a stricter threshold, which resulted in shutting in two low IRI injectors (all injectors open in Case-1). This is a

Fig. 7 Stages of Fuzzy Control

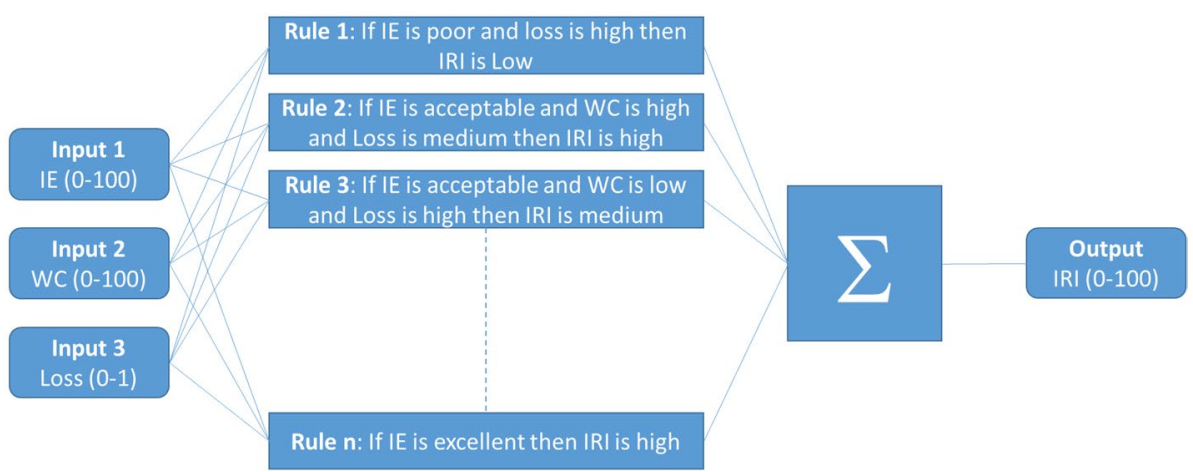



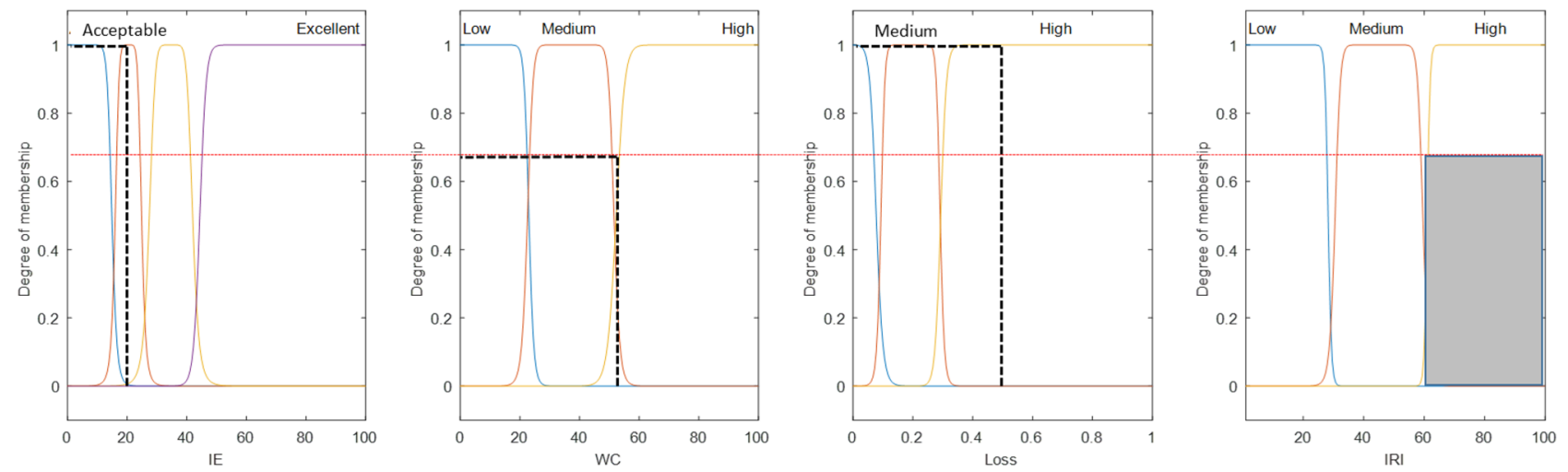

IF $\underline{\text { IE }}$ is acceptable AND $\underline{\text { WC }}$ is high AND $\underline{\text { LOSS }}$ is high THEN IRI is high
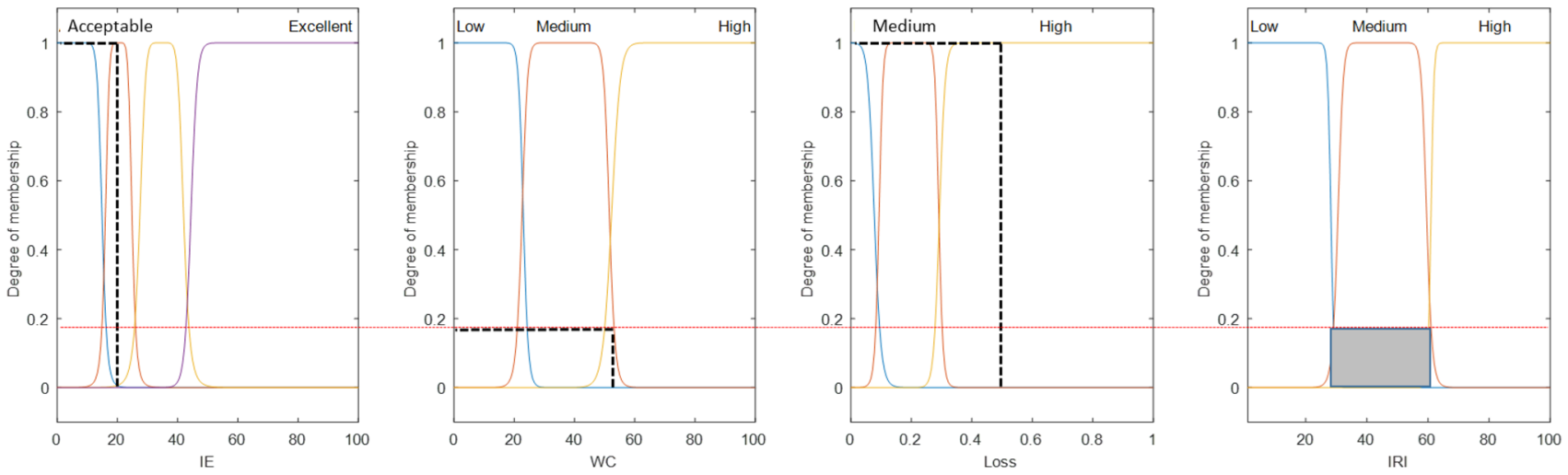

IF $\underline{\text { IE }}$ is acceptable AND $\underline{\text { WC }}$ is medium AND $\underline{\text { LOSS }}$ is high THEN IRI is medium

Fig. 9 Rule compositions with AND operator

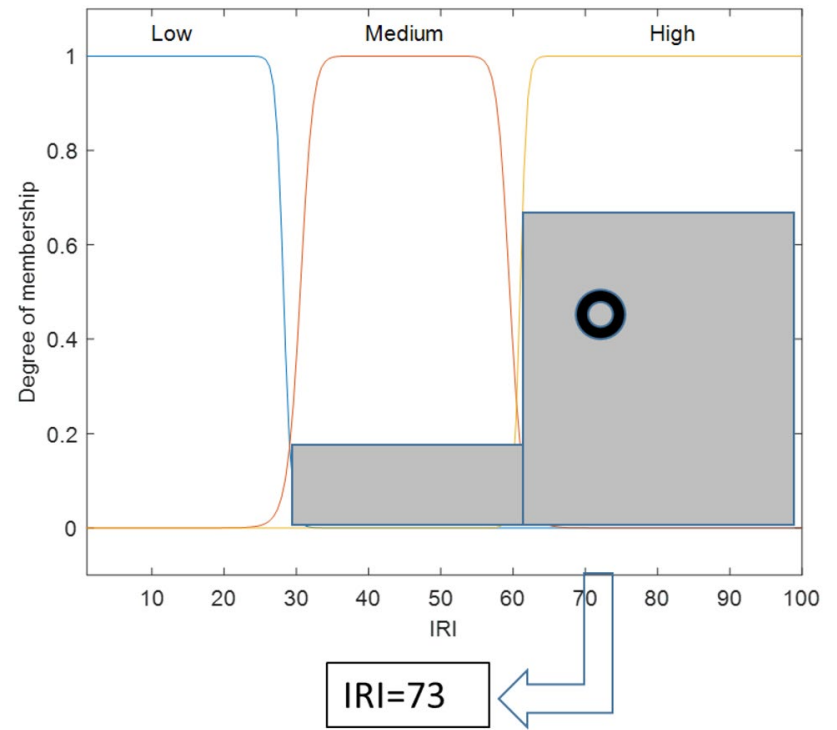

Fig. 10 Determination of IRI value using the centroid method

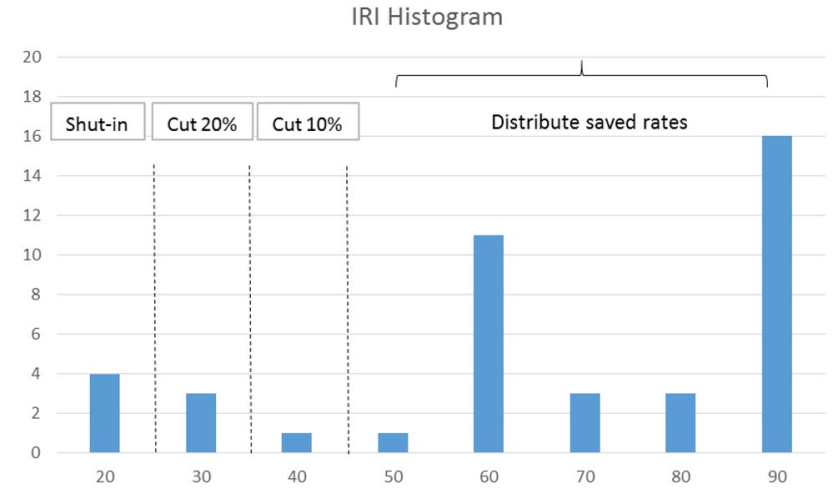

Fig. 11 An example of an IRI histogram showing how many wells were shut-in, rate-discounted, or received additional injection volume

great example of the versatility of our workflow to continue adjusting injection rates over time to reflect the changing IRI, which is one of the key enhancements to the current workflow. By further examine the objective function, the 
Fig. 12 Inj-1 injection rate comparison between cases with different assigned thresholds resulting in different injection rate adjustment in each scenario

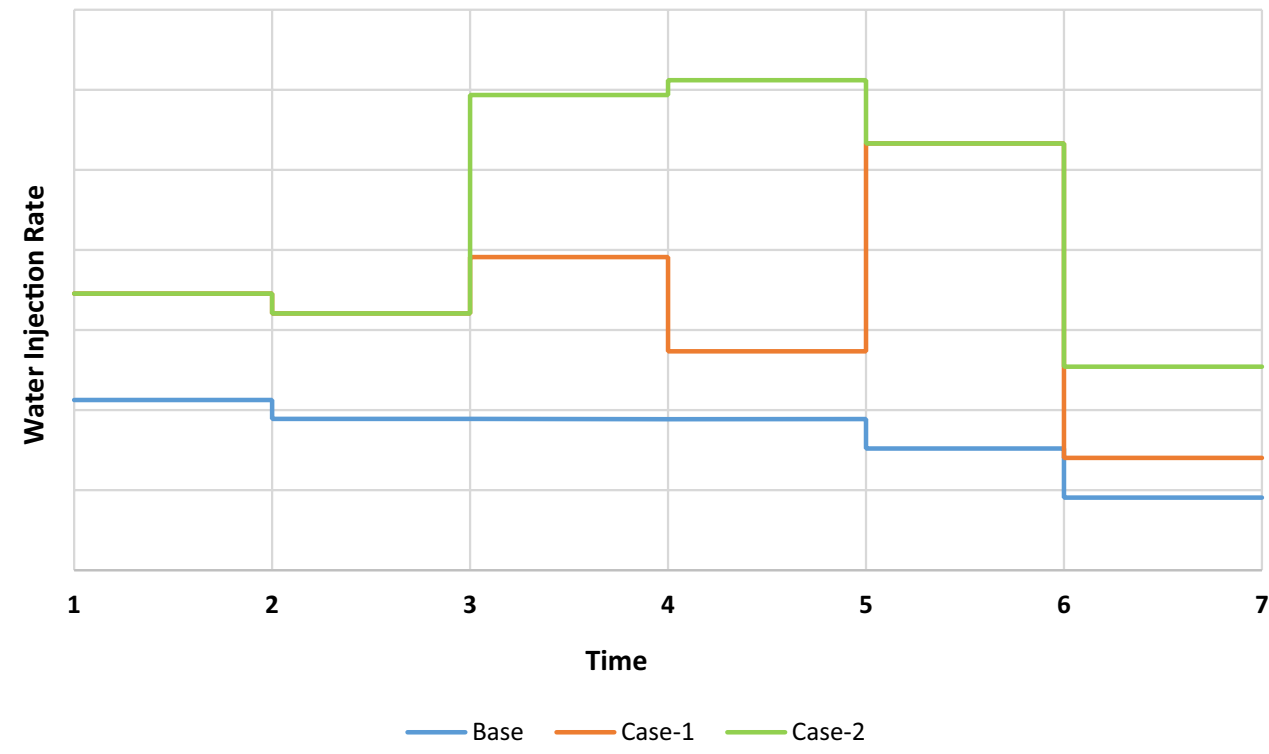

optimum case resulting in higher cumulative oil production is Case-2. Other scenarios that injected higher water volumes compared to Case-2 produced less cumulative oil and higher water cut, indicating water recycling.

Figure 13 shows oil rate comparison of high and lowperformance producers. Comparing the results from the Base Case and Case-1, the plot shows a clear and tangible oil rate enhancement in Prod-2. Looking at the Prod-1 oil rate, there is a negligible change. This trend is repeated in other similar producers in our field application resulting in a higher net cumulative oil from Case-1 compared to the Base Case. Besides the oil production gain, Fig. 14 shows the water production from Prod-2 decreased indicating that our action resulted in a more proper sweep around that producer.
Despite the change in the injection scheme, the reservoir pressure is maintained in the area as shown in Fig. 15.

For the good producer, the improvement in oil production is around $7 \%$ while water reduction is about $8 \%$. Considering the rates the well is producing at, these performance improvements are quantitatively valuable, especially when taking into account the excellent pressure maintenance too.

By further examining streamlines of injector- 1 and the producers within its irrigation region, we see that the optimum case had better sweep, more connectivity to nearby producers, and outreached more producers as depicted in Fig. 16.

Figure 17 shows the offset oil production from all producers supported by Injector-2 over multiple timesteps. It
Fig. 13 Effect of the workflow on the oil production rate from a good and weak producer

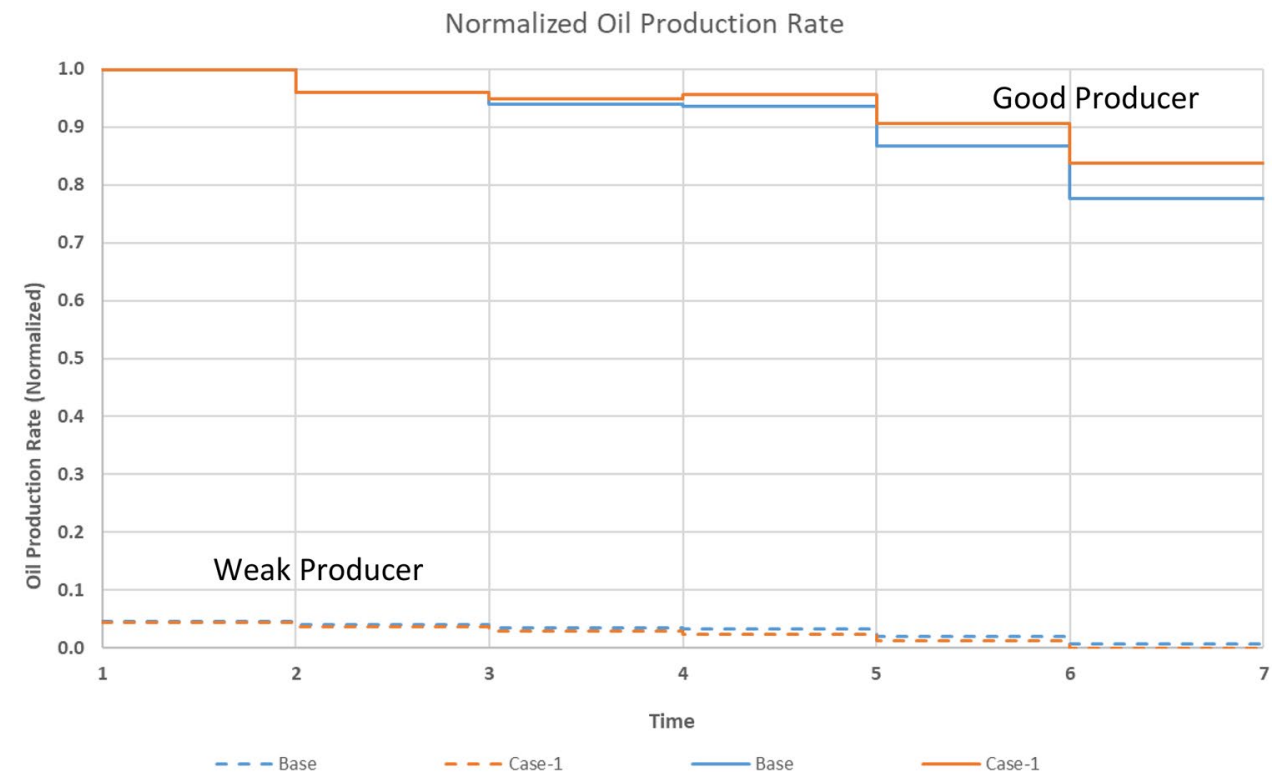


Fig. 14 Effect of the workflow on the water production rate from a good producer
Fig. 15 Area pressure comparison before and after applying the workflow
Normalized Water Production Rate

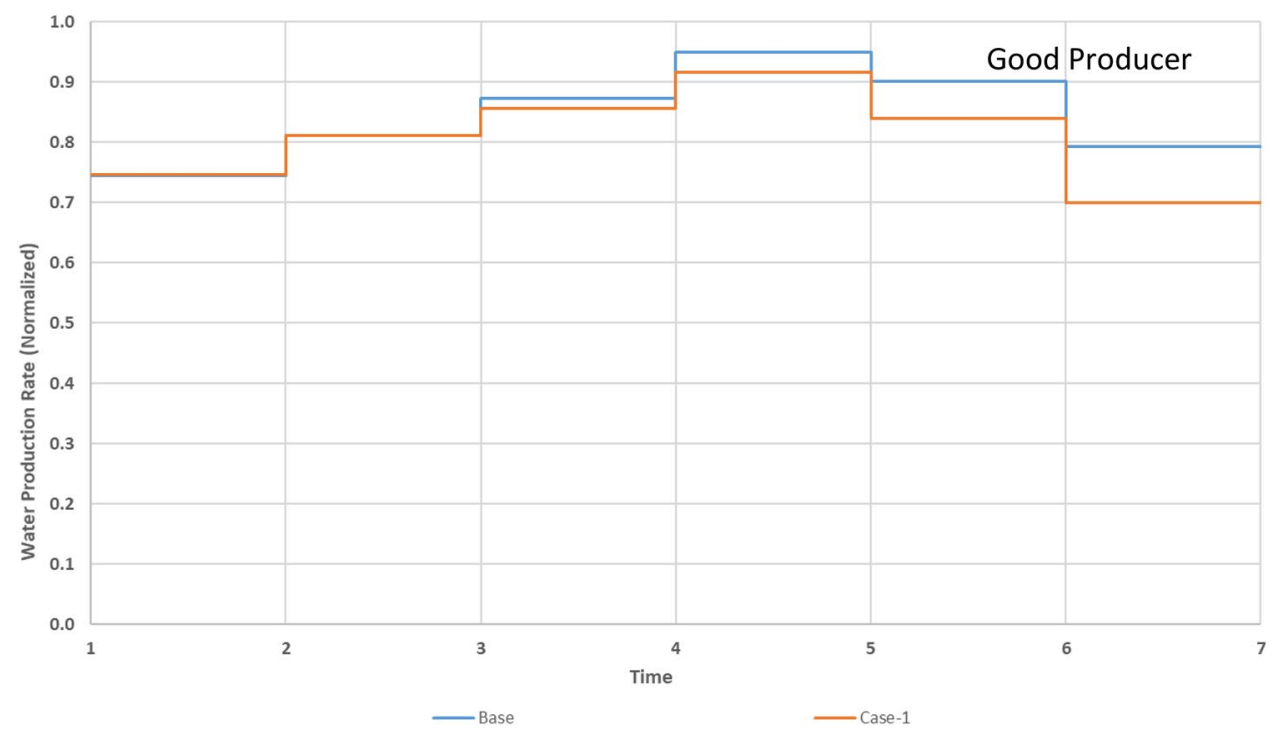

Normalized Pressure

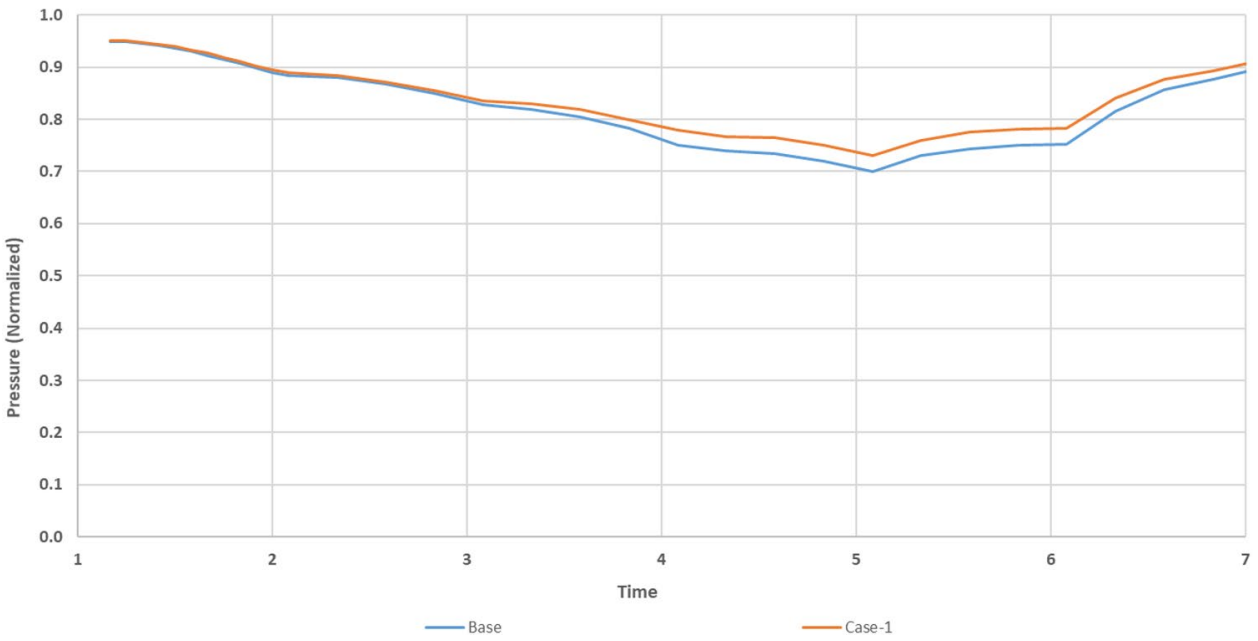

can be clearly seen that the new injection rates assigned by our workflow always yielded more offset oil production as a result of smartly redistributing injection rates.

Figure 18 is similar to Fig. 17 but adds the gain in the offset oil production rate as a result of assigning injection rate to Injector- 3 based on the weighted average method. Still, the gain from the effective IRI method is more prominent.

\section{Conclusion}

In this work, we presented a genuine approach to optimize field wide injection strategy using streamlined information coupled with fuzzy logic. The following points highlight the main features of the work presented in this paper:
- Different sensitivity runs were made to determine the best threshold and rate discount combination that optimizes our objective function.

- Accounting for the water injection capacity and IRI of each injector by using ETV are vital to proper injection optimization.

- One of the key features of the workflow is the continuous adjustment of injection rates over time to better reflect the changing IRI.

- Utilizing our versatile workflow to adjust the injection rates translated to better sweep, better pressure support, more connectivity to nearby producers and ultimately maximizing cumulative oil production. 


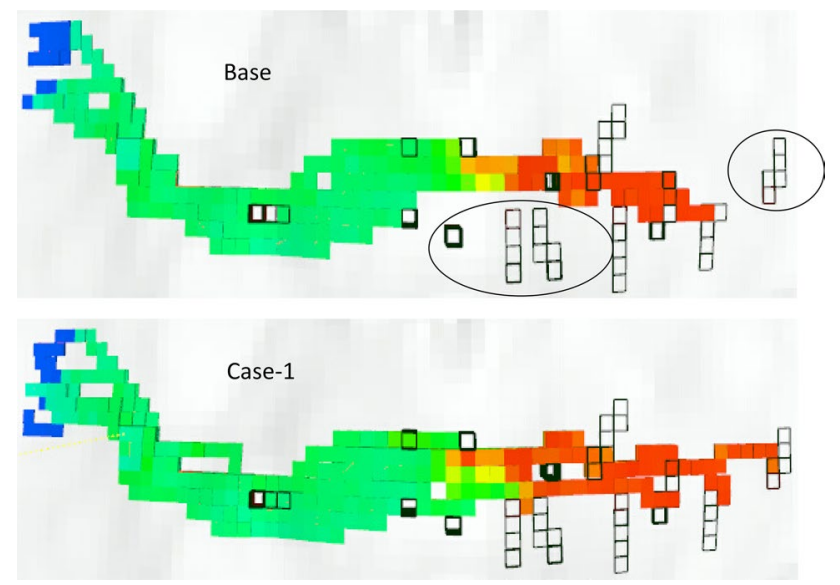

Fig. 16 An example of streamline connectivity before and after applying the workflow where cells are color-coded by water saturation (blue is high water saturation)

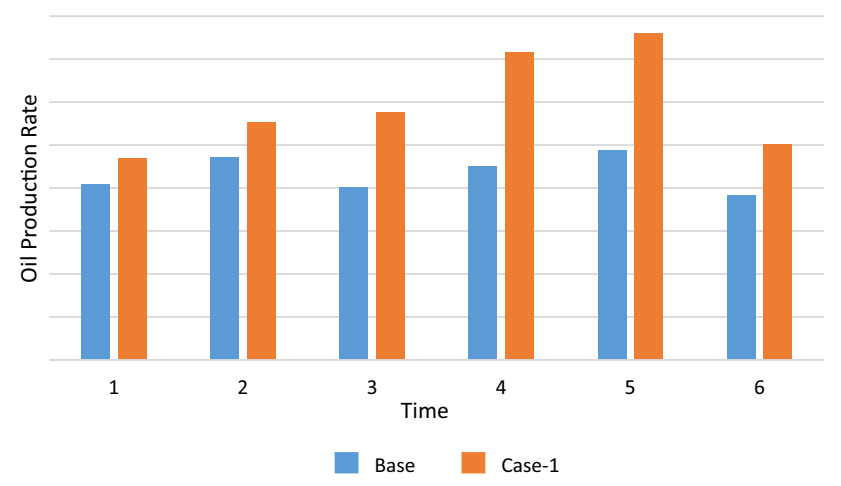

Fig. 17 Comparison of offset oil production rate from Inj-2

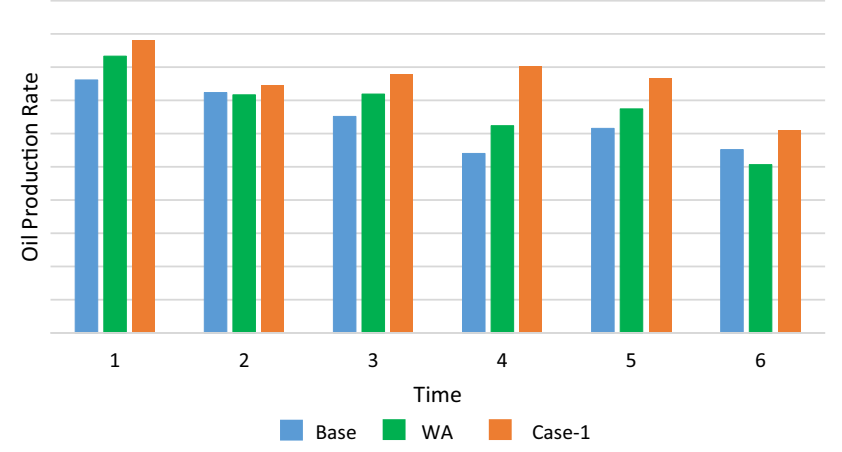

Fig. 18 Comparison of offset oil production rate between weighted average vs. ETV adjusted rates for Inj-3

- Oil rate comparison of producers showed a clear and tangible oil rate enhancement to the good producers while negligible change was noticed in the weak ones.
- The new injection rates assigned by our workflow yielded more offset oil production compared to the base case.

Funding The authors received no financial support, for the research and authorship of this article. Publication of thearticle was covered by Springer Open Membership.

\section{Declaration}

Conflict of interest On behalf of all the co-authors, the corresponding author states that there is no conflict of interest.

Open Access This article is licensed under a Creative Commons Attribution 4.0 International License, which permits use, sharing, adaptation, distribution and reproduction in any medium or format, as long as you give appropriate credit to the original author(s) and the source, provide a link to the Creative Commons licence, and indicate if changes were made. The images or other third party material in this article are included in the article's Creative Commons licence, unless indicated otherwise in a credit line to the material. If material is not included in the article's Creative Commons licence and your intended use is not permitted by statutory regulation or exceeds the permitted use, you will need to obtain permission directly from the copyright holder. To view a copy of this licence, visit http://creativecommons.org/licenses/by/4.0/.

\section{References}

Al-Zawawi AS, Hayder ME, Baddourah MA, Abd Karim, MG, Wahyu H. Using Streamline and Reservoir Simulation to Improve Waterflood Management. In: Paper presented at the SPE Middle East Oil and Gas Show and Conference, Manama, Bahrain, September 2011. https://doi.org/10.2118/141794-MS

Bouaouaja, M. T., Basioni, M., Nasiri, A. et al. 2012. Application of streamline simulation to reservoir management : management of WAG Injection Optimization. Presented at the Abu Dhabi International Petroleum Conference and Exhibition, Abu Dhabi, UAE, 11-14 November. SPE-162380-MS. https://doi.org/10.2118/ 162380-MS.

BuKhamseen, Nader Y., Al-Najem, Abdullah A., Saffar, Ali H., and Zeid M. Al-Ghareeb. "Streamline information guided by fuzzy logic to optimize field injection/production strategies." Paper presented at the SPE Kingdom of Saudi Arabia Annual Technical Symposium and Exhibition, Dammam, Saudi Arabia, April 2016. https://doi.org/10.2118/182742-MS

BuKhamseen, Nader Y., Al-Najem, Abdullah A., Saffar, Ali H., and Santiago A. Ganis. An injection optimization decision-making tool using streamline based fuzzy logic workflow. Paper presented at the SPE Reservoir Characterisation and Simulation Conference and Exhibition, Abu Dhabi, UAE, May 2017. https://doi.org/10. 2118/186021-MS

Datta-Gupta, A. and King, M.K. 2007. Streamline Simulation: Theory and Practice. Textbook Series, SPE, Richardson, Texas.

Ibrahim MN, Clark RA, Bader SA (2007) Streamline simulation for reservoir management of a super giant: sabriyah field (North Kuwait) case study. In: Paper presented at the SPE Middle East Oil and Gas Show and Conference, Manama, Bahrain, March 2007. https://doi.org/10.2118/105069-MS 
Kornberger M, Thiele MR (2014) Experiences With an Efficient RateManagement Approach for the 8th Tortonian Reservoir in the Vienna Basin. SPE Res Eval \& Eng 17:165-176. https://doi.org/ 10.2118/166393-PA

Liu, X., Wang, Z. P. and Wang, Y. 2010. Medium-high permeability mature reservoir management study: an innovative methodology and case study. Presented at the SPE international oil and gas conference and exhibition in China, Beijing, China, 8-10 June. SPE-130134-MS. https://doi.org/10.2118/130134-MS.

Meyers, P. J. 2010. Build Your Own Weighted Sort (GA Style). Moz. 14 September 2010, https://moz.com/blog/build-your-own-weigh ted-sort. Accessed 8 February 2017.

Spyrou, C. E., La Rosa, A. P., Khataniar, S. K. et al. 2017. An approach to alternative waterflood designs and operations using streamline simulation: application to an oil field in the North German Basin. Presented at the SPE Middle East Oil \& Gas Show and Conference, Manama, Bahrain. SPE-183879-MS. https://doi.org/10. 2118/183879-MS

Thiele, M. R. and Batycky, R. P. 2003. Water injection optimization using a streamline-based workflow. Presented at the SPE Annual
Technical Conference and Exhibition, Denver, Colorado, 5-8 October. SPE-84080-MS. https://doi.org/10.2118/84080-MS.

Thiele, M. R., Batycky, R. P. and Fenwick, D. H. 2010. Streamline Simulation for Modern Reservoir-Engineering Workflows. JPT 62 (1):64-70. SPE-118608-JPT. https://doi.org/10.2118/118608-JPT.

Widarsono, B., Atmoko, H., Robinson, W. IV, Yuwono, I.P., Tunggal, F.S., and Ridwan. 2005. Application of fuzzy logic for determining production allocation in commingled production wells. Paper SPE 93275 presented at the SPE Asia Pacific Oil and Gas Conference and Exhibition, Jakarta, 5-7 April. https://doi.org/10.2118/ 93275-MS.

Zadeh LA (1965) Fuzzy sets. Inf Control 8(3):338-353

Publisher's Note Springer Nature remains neutral with regard to jurisdictional claims in published maps and institutional affiliations. 